\title{
La resistividad eléctrica como parámetro de control del hormigón y de su durabilidad
}

\author{
C. Andrade ${ }^{1}$, R. D'Andrea ${ }^{2}$
}

${ }^{1}$ Centro de Investigación en Seguridad y Durabilidad de Estructuras y Materiales, CISDEM (CSIC-UPM) -España.

${ }^{2}$ Instituto Español del Cemento y sus Aplicaciones IECA- España.

\section{Información del artículo \\ DOI: \\ http://dx.doi.org/10.21041/ra.v \\ $\underline{1 i 2.8}$ \\ Artículo recibido el 25 de Noviembre de 2010, revisado bajo las políticas de publicación de la Revista ALCONPAT y aceptado el 23 de Enero de 2011. Cualquier discusión, incluyendo la réplica de los autores se publicará en el primer número del año 2012 siempre y cuando la información se reciba antes del cierre del tercer número del año 2011}

\section{2011 Alconpat Internacional}

\section{Información Legal}

Revista ALCONPAT, Año 1, No. 1, Enero Abril 2011, es una publicación cuatrimestral de la Asociación Latinoamericana de Control de Calidad, Patología y Recuperación de la Construcción, Internacional, A.C., Km. 6, antigua carretera a Progreso, Mérida Yucatán, C.P. 97310, Tel.5219997385893,

alconpat.int@gmail.com, Página Web www.alconpat.org

Editor responsable: Dr. Pedro Castro Borges. Reserva de derechos al uso exclusivo No.04 2013-011717330300-203, eISSN 2007-6835, ambos otorgados por el Instituto Nacional de Derecho de Autor. Responsable de la última actualización de este número, Unidad de Informática ALCONPAT, Ing. Elizabeth Sabido Informatica ALCONPAT, Ing. Elizabeth Sabido Maldonado, Km. 6, antigua carretera a Progreso,
Mérida Yucatán, C.P. 97310 , fecha de publicación: 30 de Mayo de 2011

Las opiniones expresadas por los autores no necesariamente reflejan la postura del editor. Queda totalmente prohibida la reproducción total o parcial de los contenidos e imágenes de la publicación sin previa autorización de la ALCONPAT Internacional A.C.

\section{RESUMEN}

La necesidad de calcular la durabilidad del hormigón ha llevado a buscar un ensayo que fuera capaz de cuantificar todas las fases del proceso desde la fabricación del hormigón hasta su curado y endurecimiento. La resistividad eléctrica como inversa de la conductividaddifusividad es una propiedad del hormigón que permite su control de forma no destructiva y por ello, accesible al autocontrol. La resistividad es un indicador del fraguado y de la resistencia mecánica, del grado de saturación del hormigón y por ello del grado de curado y de la impermeabilidad o resistencia al ingreso de sustancias agresivas en el hormigón. Finalmente tiene una relación directa con la velocidad de corrosión al indicar el grado de humedad del hormigón. Por todo ello es un parámetro que permite relacionar al microestructura con el comportamiento durable del hormigón. En el artículo se describen los fundamentos de sus posibilidades.

Palabras clave: resistividad eléctrica; hormigón; durabilidad; corrosión.

\section{ABSTRACT}

The need to estimate the durability of concrete has led to the search for a test able to analyze and quantify all of the phases of the process, including the production of concrete, the curing and the hardening. The electric resistivity, as the inverse of the conductivity-diffusivity, is a property of concrete that allows its non destructive monitoring, and is thus accessible to selfcontrol. The resistivity is an indicator of the setting and the mechanical strength, of the degree of saturation of the concrete, hence of the degree of curing and of the permeability or resistance to the ingress of aggressive substances. It is also directly related to the velocity of corrosion as it indicates the degree of humidity of the concrete. Due to the above, the resistivity is a parameter that allows to correlate the microstructure to the performance of durable concrete; this paper describes the fundamentals and its possibilities

Key words: electric resistitivy; concrete; durability; corrosión.

Autor de contacto: Carmen Andrade (andrade@ietcc.csic.es) 
Revista ALCONPAT, Volumen 1, Número 2, Mayo - Agosto 2011, Páginas 90 - 98

\section{INTRODUCCIÓN}

El hormigón armado es un material muy durable en la mayoría de los ambientes pero en medios contaminados con cloruros o cuando las edades exceden 50 años, se puede producir la corrosión de su armadura. La demanda de durabilidad es pues actualmente creciente una vez que la resistencia mecánica ha sido bien diseñada y controlada. El diseño de un hormigón durable no es una tarea sencilla si se mantiene el criterio de economía máxima ya que cualquier método de protección adicional de las armaduras resulta en un incremento de costos apreciable. Por ello se sigue trabajando en la búsqueda de hormigones no permeables y cuyas propiedades de durabilidad se puedan determinar de forma rápida y sencilla.

En los últimos años se ha tratado de relacionar determinados métodos de ensayo del hormigón que miden sus propiedades de transporte con su durabilidad. Es de destacar el libro que resume el trabajo del Comité de RILEM TC-116 que fue dirigido primero por H.K. Hilsdorf y luego por J. Kropp (Kropp, 1995). En él se describen los métodos más conocidos de medida de la permeabilidad del hormigón y se valora su relación con la durabilidad, concluyendo que solo los métodos que directamente miden el transporte de cloruros o el del dióxido de carbono son realistas, aunque su uso para predecir el comportamiento a largo plazo sigue siendo una cuestión no resuelta.

Para responder a la necesidad del fabricante de hormigón y del usuario, lo idóneo es poder disponer de un ensayo no destructivo que permita el autocontrol del fabricante, así como el seguimiento del material por parte del usuario a lo largo de su ciclo de vida. Los métodos de medida de la difusión de cloruros y de carbonatación no resultan fácilmente accesibles para los fabricantes y su interpretación exige de especialistas (Page et al, 1981; Tuutti, 1982; McCarter y Garvin, 1989; Monfore, 1968; Polder et al, 2000; Whiting, 1981). Hasta el presente este tipo de ensayos "directos" de medida de la penetración en el hormigón de la sustancia agresiva son bastante usados por laboratorios especializados, que incluso realizan predicciones de vida útil basados en resultados a corto plazo, pero en cualquier caso sigue necesitándose un ensayo de control de calidad que permita una estimación de la durabilidad futura (Baroguel-Bouny, 2004).

Este tipo de ensayo no destructivo se ha publicado ya por parte de los autores que puede ser la medida de la resistividad eléctrica del hormigón (Andrade et al, 2000; Andrade y D'Andrea, 2008). Esta medida puede además valorar los siguientes aspectos del hormigón: a) El endurecimiento en estado fresco, b) El grado de curado, c) La resistencia a la penetración de los cloruros y la carbonatación y d) La velocidad de corrosión de la armadura. Resulta pues muy atractivo estudiar más a fondo sus posibilidades para tratar de conseguir un ensayo que permita una valoración de la calidad del hormigón en todos sus aspectos. La Resistividad eléctrica, $\rho$, es capaz de informar al fabricante y al usuario de la microestructura del hormigón, de su porosidad y de su contenido en agua. Con ello se tiene un control completo de las etapas por las que pasa el material. Al ser un ensayo no destructivo que se puede repetir muchas veces sin perturbar el material, es muy adecuado para el autocontrol del fabricante y para el control de calidad del usuario.

En el presente trabajo se resumen los fundamentos de la aplicación de esta medida a los distintos aspectos mencionados y además se comenta una metodología para el diseño de la mezcla de componentes del hormigón para alcanzar una resistividad determinada. 
Revista ALCONPAT, Volumen 1, Número 2, Mayo - Agosto 2011, Páginas 90 - 98

\section{QUE ES LA RESISTIVIDAD ELECTRICA}

Como indica la Ley de Ohm la resistividad es una propiedad volumétrica del material e indica su resistencia al paso de las cargas eléctricas:

$$
R=\frac{V}{I}=\rho \frac{l}{A}
$$

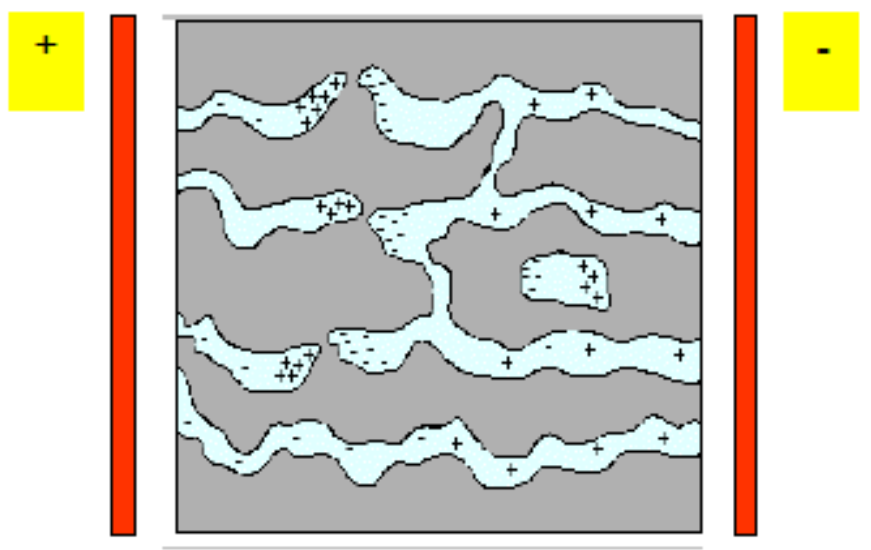

Figura 1. La porosidad se puede evaluar por la resistencia al movimiento de las cargas eléctricas

(los iones) de la fase acuosa de los poros del hormigón

Donde $\mathrm{R}$ es la resistencia eléctrica que se mide aplicando un voltaje $\mathrm{V}$ y midiendo el paso de corriente I. Esa relación es igual a la resistividad por el factor geométrico $1 / \mathrm{A}$, es decir por la $\mathrm{l}=$ distancia entre electrodos y A= área transversal de paso de la corriente.

Cuanto mayor es la resistividad menor es la porosidad del hormigón y mayor su resistencia mecánica, al tener más fase sólida por volumen. Además si el hormigón no está saturado de agua la resistividad crece, por lo que es un indicador de su grado de saturación. Por tanto, la resistividad es un indicador de la calidad del hormigón al indicar su porosidad, y es un indicador de su grado de saturación y por tanto puede servir para el control del grado de curado.

\section{LA MEDIDA DE LA RESISTIVIDAD}

Puede realizarse colocando unos electrodos en las caras paralelas de las probetas cilíndricas o usando el "método de las 4 puntas" como indican las figuras 2 y 3 adjuntas. Para la medida rápida de la Resistividad en el hormigón e in situ se ha desarrollado entre el IETcc y Geocisa un Resistivimetro portátil que se muestra en la figura 4.

Existe además una Norma UNE que describe los dos métodos de medida PNE 83988 Durabilidad del hormigón - Determinación de la resistividad del hormigón - Parte I (Método directo) y Parte II (Método de Wenner) (Wenner, 1915/16). 
Revista ALCONPAT, Volumen 1, Número 2, Mayo - Agosto 2011, Páginas 90 - 98

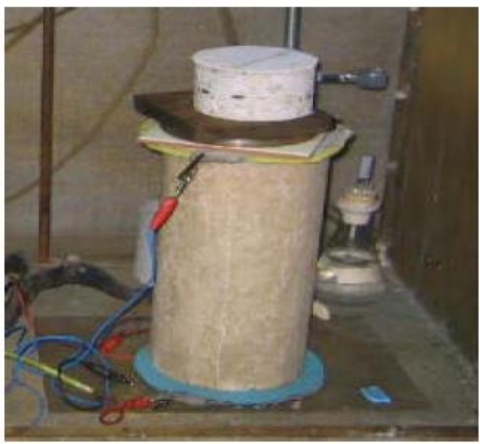

Figura 2. Método directo

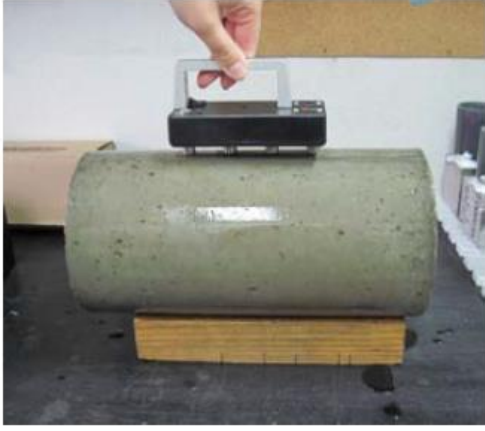

Figura 3. Método de las 4 puntas

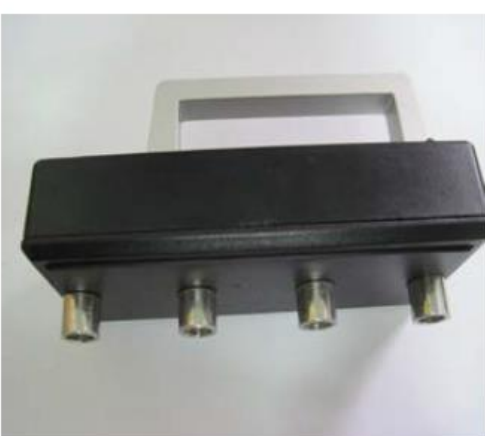

Figura 4. Resistivímetro portátil

\section{EL ENDURECIMIENTO EN ESTADO FRESCO}

Dado que al mezclar el cemento con el agua se obtiene una suspensión, la Resistividad al principio del mezclado es muy baja pero, según va hidratándose el cemento y el hormigón va fraguando y endureciendo, la resistividad aumenta. En la figura 5 se muestra un ejemplo de la curva de resistividad durante las primeras $24 \mathrm{~h}$. El desarrollo de la curva permite identificar la acción de los aditivos, así como otros aspectos del fraguado (Andrade y Rebolledo, 2011).

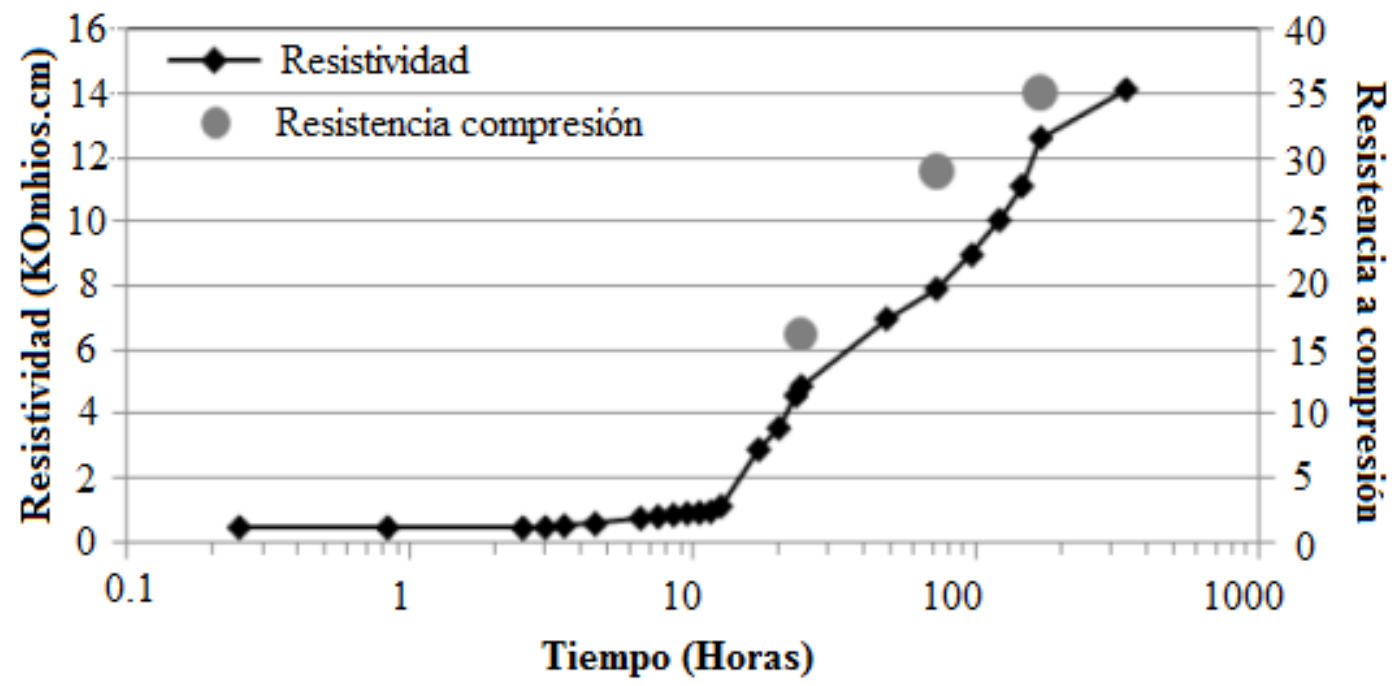

Figura 5. Desarrollo de la resistividad durante el fraguado y endurecimiento

La relación con la resistencia a compresión que se indica en esta figura 5 también está siendo objeto de estudio (Andrade et al, 2009) y se están desarrollando expresiones matemáticas que ligan ambos parámetros, lo que permitiría al fabricante predecir la resistencia mecánica desde las primeras horas. 


\section{Rotura \& Resistividad}

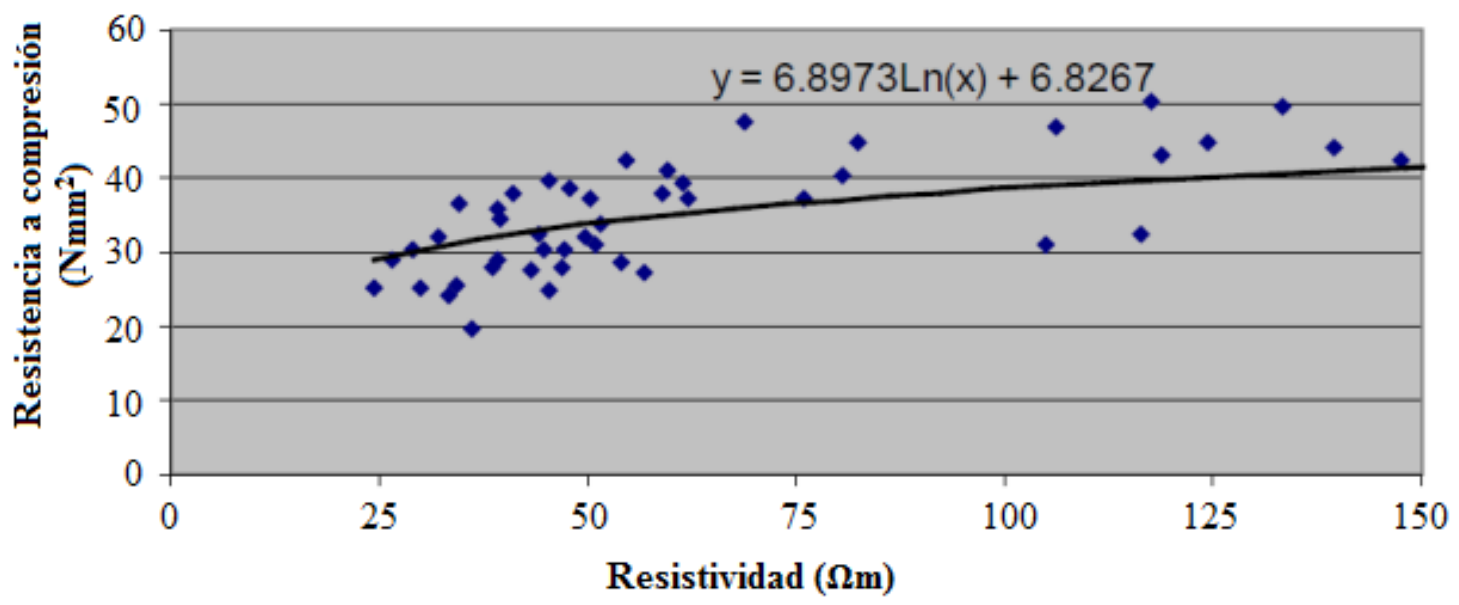

Figura 6. Relación entre resistencia a compresión y resistividad para un tipo de cemento.

La evolución de la resistividad es paralela a la de la resistencia como se muestra en la figura 6 y por ello la resistividad permite además de predecir la resistencia, indicar el "factor de edad" que es esencial para los modelos de durabilidad que se comentaran a continuación. La figura 7 muestra la inversa de la resistividad con el tiempo para un hormigón de la que se puede calcular el factor de edad q que se indica en la ecuación (2).

$$
\rho(t)=\rho_{0}\left(t / t_{0}\right)^{+q}
$$

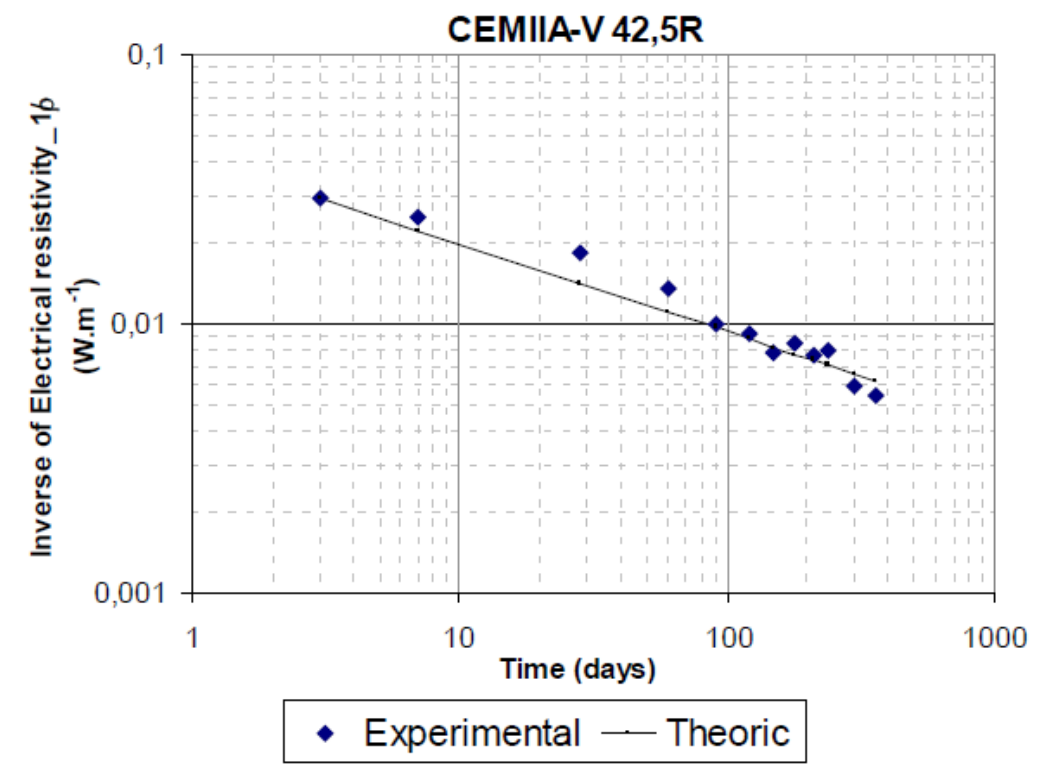

Figura 7. Variación con el tiempo de la inversa de la resistividad (conductividad)

\section{EL GRADO DE CURADO DEL HORMIGÓN}

El curado del hormigón es un aspecto muy importante que afecta al comportamiento a largo plazo si bien no hay un método que permita cuantificarlo. La resistividad si lo permite ya que detecta muy bien el secado superficial, y con ello el grado de evaporación o saturación de los poros del hormigón. Comparando la resistividad de un hormigón bien curado con otro mal curado es 
Revista ALCONPAT, Volumen 1, Número 2, Mayo - Agosto 2011, Páginas 90 - 98

posible encontrar un porcentaje de saturación relativa de los poros que cuantifica el grado de curado (figura 8). Esta relación obtenida en (Fernández, 2008) resulta un método muy sencillo de control en obra del curado.

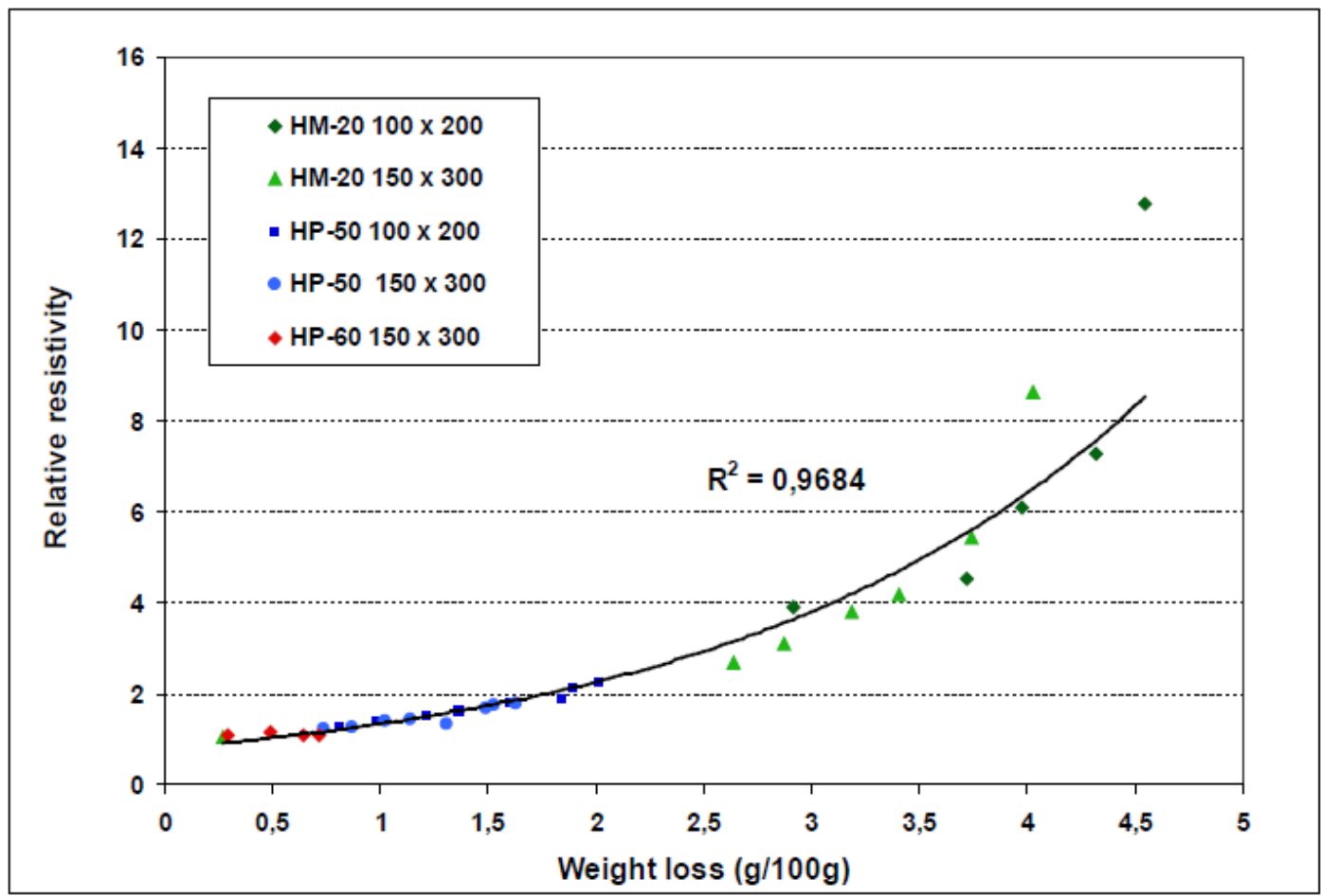

Figura 8. Resistividad relativa frente a pérdida de peso por secado en el curado

\section{MODELOS DE DURABILIDAD: LA RESISTENCIA A LA PENETRACIÓN DE LOS CLORUROS Y LA CARBONATACIÓN. LA VELOCIDAD DE CORROSIÓN DE LA ARMADURA}

La resistividad también puede ser utilizada en una expresión matemática para la predicción tanto del periodo de iniciación hasta que se desarrolla la corrosión de la armadura como para cuantificar el periodo de propagación de la corrosión (Andrade et al, 2000; Andrade y D'Andrea, 2008). Ésta posibilidad se basa en la relación inversa entre la resistividad eléctrica y la difusividad de los iones. Es decir, a mayor resistividad se produce un movimiento menor de las cargas eléctricas (los iones de los poros del hormigón) debido a que mayor resistividad indica menor porosidad.

Si la vida total del la estructura es la suma del periodo de iniciación hasta que la armadura se corroe más el periodo de propagación de la corrosión hasta alcanzar un determinado ancho de fisura del recubrimiento, se puede decir que el tiempo de vida total será: $t=t i+$ tp. Y si consideramos que el periodo de iniciación es proporcional a la raíz cuadrada del tiempo y el de propagación es lineal con la perdida de diámetro de la armadura resulta:

$$
t=V_{C l, \mathrm{CO}_{2}} \sqrt{t_{i}}+\frac{P_{x}}{V_{c o r r}}
$$


Revista ALCONPAT, Volumen 1, Número 2, Mayo - Agosto 2011, Páginas 90 - 98

Sustituyendo en esta expresión el valor de la velocidad $\mathrm{V}_{\mathrm{Cl}}, \mathrm{CO} 2$ de ingreso de los cloruros o la carbonatación por su equivalente de resistividad eléctrica la expresión que se obtiene es:

$$
t_{l}=t_{i}+t_{p}=\frac{x^{2} \rho_{e s} r_{C l, \mathrm{CO}_{2}}}{F_{\mathrm{Cl}, \mathrm{CO}_{2}}}
$$

En esta expresión se incluye el "Factor de Reacción" que depende del tipo de cemento y los factores $\mathrm{F}_{\mathrm{Cl}}, \mathrm{CO} 2$ que depende del tipo de ambiente. De esta manera el cálculo de la vida útil es posible a partir del valor de la resistividad del hormigón y de unos factores que tienen unos valores fijos que dependen del ambiente o del tipo de cemento.

Por ejemplo, para un recubrimiento de $5 \mathrm{~cm}$ en un hormigón a colocar en la clase de ambiente IIIb fabricado con un cemento tipo II/A, para una vida útil de 100 años y los factores de reacción y de edad que se indican en la tabla 1, los cálculos con la formula (4) resultan en un valor de la resistividad necesario de $87,6 \Omega \mathrm{m}$.

Tabla 1. Ejemplo de cálculo de la resistividad necesaria

\begin{tabular}{|c|c|}
\hline Tipo de Cemento II/A & $\mathrm{r}_{\mathrm{Cl}}=1,8$ \\
Clase de Expocisión (XS2) & $\mathrm{F}\left(\mathrm{cm}^{3} / \mathrm{año}\right)=17000$ \\
Tiempo de vida & $\mathrm{t}(\mathrm{años})=100$ \\
Profundidad de Recubrimiento & $\mathrm{X}_{\mathrm{Cl}}(\mathrm{cm})=5$ \\
Factor de edad durante 10 años & $\mathrm{q}=0.3$ \\
\hline
\end{tabular}

$$
5=\sqrt{\frac{17000}{\rho_{0}\left(\frac{10}{0,0767}\right)^{0,3} \cdot 1,8}} \cdot \sqrt{100}\left\{\rho_{0}(\Omega \cdot \mathrm{cm})=8760 \rightarrow \rho_{0}(\Omega \cdot m)=87,6\right.
$$

Es decir, será necesario diseñar una mezcla de hormigón que dé a 28 días de curado húmedo esa resistividad. Así, la durabilidad es posible calcularla con conceptos simples mediante la medida de la resistividad del hormigón a 28 dias de forma paralela a como se ensaya la resistencia a compresión.

En cuanto a la velocidad de corrosión de la armadura, es también proporcional al grado de humedad y por tanto a la resistividad del hormigón en el ambiente en que se encuentre: $\mathrm{I}_{\text {corr }}=$ $\mathrm{k}_{\text {corr }} / \rho$ donde $\mathrm{k}_{\text {corr }}$ es una constante. El grado de humedad del hormigón depende del clima, es decir de la cantidad de lluvia y de la temperatura. Con esta relación es posible calcular lo que duraría el periodo de propagación de la corrosión hasta que se alcance una fisuración prefijada del recubrimiento.

\section{DISEÑO DE LA MEZCLA DE HORMIGON PARA OBTENER UNA DETERMINADA RESISTIVIDAD}

Este aspecto es el que permite usar el concepto de la resistividad de una forma práctica por el fabricante, ya que es necesario diseñar una mezcla que aporte los valores requeridos de la misma manera que se hace para conseguir una determinada resistencia mecánica. Es posible relacionar la resistividad con la composición de la mezcla a través de las Leyes de Powers (que calculan la 
Revista ALCONPAT, Volumen 1, Número 2, Mayo - Agosto 2011, Páginas 90 - 98

porosidad de la pasta de cemento a partir de la relación a/c) y de Archie (que relaciona la porosidad con la resistividad).

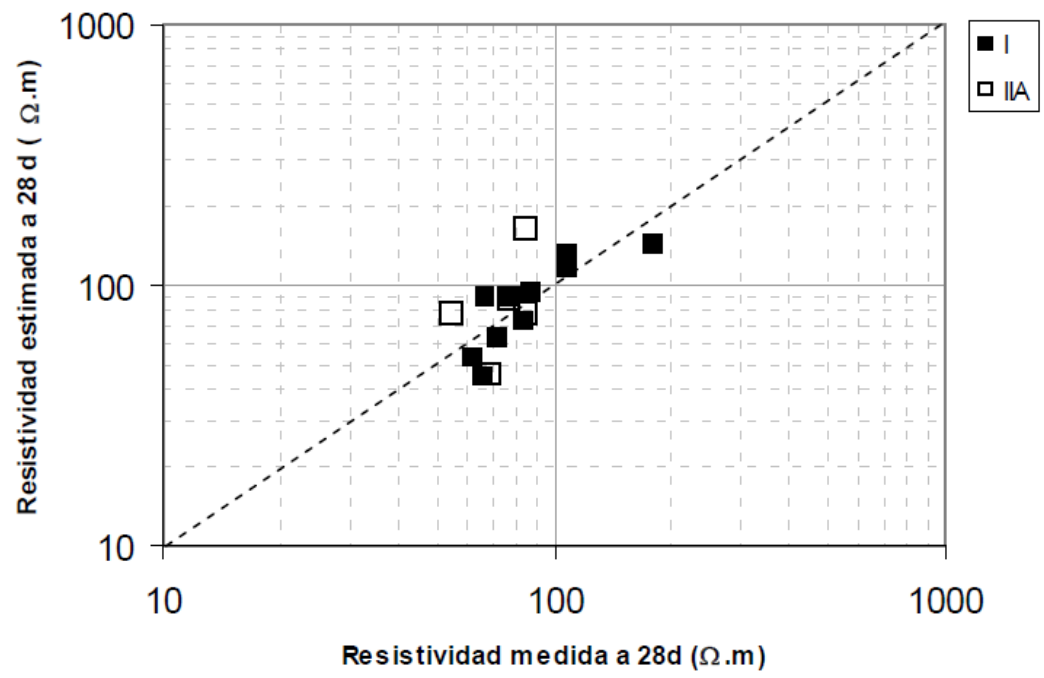

Figura 9. Relación entre los valores de la resistividad, medidos a los 28 días y estimados a partir del modelo basado en la ley de Archie

Así, suponiendo un contenido en cemento por m3 de hormigón, es decir un volumen de pasta por $\mathrm{m} 3$, se puede calcular la relación a/c que permite obtener una determinada porosidad y con ello una determinada resistividad del hormigón. Para completar el estudio es necesario también aplicar el "factor de tortuosidad" que se ha calculado para cada tipo de cemento. En la figura 9 se dan las relaciones encontradas en una serie de hormigones estudiados entre resistividad eléctrica calculada y medida. La metodología que se puede seguir es:

$\left.\mathbf{1}^{\mathbf{0}}\right)$ Se elige el tipo de cemento, y se obtendrá del fabricante de cementos los valores de las variables factor de retardo (r) y tortuosidad $(\tau)$.

$\left.\mathbf{2}^{\circ}\right)$ Se estima una relación $w / c$, que será introducida en el cálculo de la porosidad de la pasta por la ecuación de Powers, $y$ un contenido de cemento $\boldsymbol{c}$, que será introducido en el cálculo de la porosidad del hormigón, donde $\varepsilon=\varepsilon_{\mathrm{p}} * \gamma$.

$3^{\circ}$ ) Se aplica la ecuación basada en la ley de Archie, $\rho=\rho_{o} \cdot\left(\varepsilon_{p} \cdot \gamma\right)^{-\tau}$ donde se comprueba que los valores estimados para w/c y c son los ideales para alcanzar $\rho$ calculado en el paso 2. En el caso de que no se logre el objetivo se pasaría a un paso complementario que sería, o bien elegir otro tipo de cemento con otros valores de $\mathbf{r}$ y $\tau$ hasta comprobar la validez de $\mathrm{w} / \mathrm{c}$ y c, o bien modificar los valores de w/c y c hasta lograrlo.

$\left.4^{\circ}\right)$ Finalmente, se comprueba que el valor de $\rho_{a p}$ cumple el demandado por el proyectista del proyecto mediante la determinación experimental de la resistividad en el hormigón saturado a los 28 días, $\rho_{\mathrm{es}}$, a partir de la relación $\rho_{\mathrm{ap}} / \mathrm{r}$.

\section{REFERENCIAS}

Kropp, J. H. H. K. (1995). "Performance criteria for concrete durability", RILEM REPORT $\mathrm{n}^{\circ}$ 12: 1-328.

Page, C.L., Short, N.R. \& El Tarras, A. (1981) "Diffusion of Chloride lons In Hardened Cement Pastes", Cement and Concrete Research. Vol. 1 1, No. 3, pp. 395-406.

Tuutti, K. (1982) "Corrosion of Steel in Concrete", Swedish Cement and Concrete Research Institute (CBI) no.4-82, Stockholm. 
Revista ALCONPAT, Volumen 1, Número 2, Mayo - Agosto 2011, Páginas 90 - 98

McCarter, W. J., and Garvin, S., (1989) "Dependence of Electrical Impedance of Cement-Based Materials on their Moisture Condition", J. Phys. D: Appl. Phys., Vol. 22, pp. 1773 - 6

Monfore, G.E. (1968) "The electrical resistivity of concrete", Journal of PCA, pp.35-48.

Polder, R.; Andrade, C.; Elsener, B.; Vennesland, O.; Gulikers, J.; Weidert, R.; and Raupach, M.; "Test methods for onsite measurement of resistivity of concrete", RILEM TC 154-EMC: Electrochemical techniques for measuring metallic corrosion, Materials and Structure, Vol 33 (2000), pp. 603-611.

Whiting, D. 1981, "Rapid determination of the chloride permeability of concrete", Federal Highway Administration - Report FHWA/RD-81/119.

Baroguel-Bouny, V.; et al., "Concrete design for a given structure service life. Durability control with respect to reinforcement corrosion and alkali-silica reaction. State-of-the-art and guide for the implementation of a perfomance-type and predictive approach based upon durability indicators" (in French), Scientific and technical documents-AFGC, July 2004, 1-252.

Andrade, C., Alonso, C., Arteaga, A. \& Tanner, P. (2000) "Methodology based on the electrical resistivity for the calculation of reinforcement service life", 5th Canmet/ACI Int. Conference on Durability of concrete - Supplementary papers volume. Barcelona, Spain, pp. 899-915.

Andrade, C. and R. d'Andrea (2008). "Electrical resistivity as microstructural parameter for the calculation of reinforcement service life", Microstructure Related Durability of Cementitious Composites, Vols 1 and 2. W. Sun, K. VanBreugel, C. Miao, G. Ye and H. Chen. 61: 1483-1490. Wenner F., "A Method of Measuring Earth Resistivity", Bull, National Bureau of Standards, Bull 12(4) 258, s 478-496; 1915/16.

Andrade C, Rebolledo, N. (2011). Monitoring by resistivity and ultrasonic velocity of the effect of the $w / c$ ratio on setting and hydration evolution, XIII International Congress on teh Chemistry of Cement, Madrid.

Andrade C., d'Andréa R., Castillo A., Castellote M. (2009), The use of electrical resistivity as ndt method for specification the durability of reinforced concrete NDTCE'09, Non-Destructive Testing in Civil Engineering, Nantes, France, June 30th - July $3^{\text {rd }}$.

Fernandez Luco L. (2008) Valoración de técnicas no destructivas para el control de la eficiencia de curado del hormigón. Tesis Doctoral. Universidad de Alicante, Septiembre/2008. 